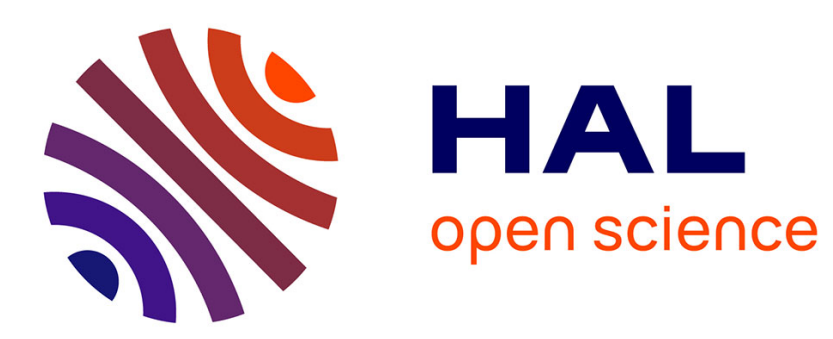

\title{
A Modeling Framework for Resource Service Sharing in a Cloud Manufacturing System
}

\author{
Yongkui Liu, Xun Xu, Lin Zhang, Fei Tao
}

\section{To cite this version:}

Yongkui Liu, Xun Xu, Lin Zhang, Fei Tao. A Modeling Framework for Resource Service Sharing in a Cloud Manufacturing System. IFIP International Conference on Advances in Production Management Systems (APMS), Sep 2015, Tokyo, Japan. pp.412-419, 10.1007/978-3-319-22759-7_48 . hal-01431124

\section{HAL Id: hal-01431124 \\ https://hal.inria.fr/hal-01431124}

Submitted on 10 Jan 2017

HAL is a multi-disciplinary open access archive for the deposit and dissemination of scientific research documents, whether they are published or not. The documents may come from teaching and research institutions in France or abroad, or from public or private research centers.
L'archive ouverte pluridisciplinaire HAL, est destinée au dépôt et à la diffusion de documents scientifiques de niveau recherche, publiés ou non, émanant des établissements d'enseignement et de recherche français ou étrangers, des laboratoires publics ou privés. 


\title{
A Modeling Framework for Resource Service Sharing in a Cloud Manufacturing System
}

\author{
Yongkui $\mathrm{Liu}^{1,2,3}$, Xun $\mathrm{Xu}^{1}$ Lin Zhang ${ }^{2}$, and Fei Tao ${ }^{2}$ \\ 1 Department of Mechanical Engineering, The University of Auckland, Auckland, \\ New Zealand \\ 2 School of Automation Science and Electrical Engineering, Beihang University, \\ Beijing 100191, China \\ ${ }^{3}$ Center for Complex Systems, School of Mechano-Electronic Engineering, Xidian \\ University, Xi'an 710071, China
}

\begin{abstract}
Cloud manufacturing (CMfg) is a novel business paradigm with resource service (RS) sharing being one of the most important purposes. From the perspective of enterprise business interactions, how to achieve full RS sharing is an important research issue in CMfg as it plays a critical role in enhancing the performance and efficiency of a CMfg system. However, the research on CMfg RS sharing is challenged by the complexity of a CMfg system, mainly coming from the vast number of involved enterprises and their complex business interactions. In this paper, we propose a modeling framework for CMfg RS sharing by regarding a CMfg system as a complex system and elaborating the problem from the perspective of complex systems modeling and simulation. We summarize systematically the factors that need to be considered for building a system of CMfg RS sharing, which is followed by the modeling procedure.
\end{abstract}

Keywords: cloud manufacturing, complex system, modeling framework, resource service sharing

\section{Introduction}

CMfg is a new manufacturing business paradigm aiming to achieve large-scale resource service (RS) sharing and efficient enterprise collaboration [1,2]. The extent and effect of CMfg RS, which depend on both technological and business factors, to a great extent, determines the performance and efficiency of a CMfg system.

CMfg, through building a common platform and the aggregation of largescale manufacturing services, provides an excellent support for RS sharing. Through the CMfg platform, any two enterprises can establish business relations and share resources and services. In addition, CMfg provides a good support for various forms of enterprise collaboration (such as random one-off trades, virtual enterprise, extended enterprise) through building a flexible service sequence. Hence, a CMfg system is a highly dynamic system with constant changing cooperation 
relationship between enterprises. More importantly, each enterprise is an adaptive entity in terms of the adaptive decision-making on RS sharing according to internal (e.g., resource utilization, revenue) and external conditions (e.g., supply and demand of resources). All of those make a CMfg system a complex adaptive system.

Currently, there is rare work on CMfg RS sharing and the related research is far from enough in revealing CMfg RS sharing methods and mechanisms. In this aspect, complex systems modeling and simulation provide an effective means. Though building a quantitative mathematical model of CMfg RS sharing, we can simulate the RS sharing process systematically and accurately. In this paper, we propose a modeling framework for CMfg RS sharing by comprehensively summarizing the relevant factors, and presenting the modeling procedure.

\section{Literature review}

Li et al. [1] first systematically proposed the concept of CMfg. Thereafter, CMfg received much attention from researchers and practitioners. He and Xu [3] provided a comprehensive summary of existing architecture and frameworks of $\mathrm{CMfg}$, as well as the latest research advancement on CMfg key technologies and service management. Xu [4] suggested two types of cloud computing adoptions in the manufacturing sector, manufacturing with direct adoption of cloud computing technologies and CMfg. Tao et al. [5] studied utility modeling, equilibrium and coordination of demanders, providers and an operator. Tai et al. [6] analyzed the issue of CMfg enterprise cooperation. Cloud service trading model and trading flow [7] have also been investigated.

Resource, capability and demand sharing, enterprise collaboration, enterprise network as important research issues have been studied. Renna and Argoneto [8] proposed a game theory coordination mechanism for the capability sharing in a network of independent plants. Yoon and Nof [9] addressed the demand and capability sharing issue in a collaborative network of independent enterprises. Two collaboration modes, i.e., partial collaboration and complete collaboration have been comparatively studied. Argoneto and Renna [10] investigated capacity sharing in CMfg by proposing a framework for capability sharing in CMfg. Moghaddam and Nof [11] investigated the demand and capability sharing issue in a collaborative network of independent enterprises with dynamic best matching of supply enterprises and customers. Jagdev and Thoben [12] discussed enterprise collaborations, identified the key types of collaborations (supply chain, extended enterprise and virtual enterprise), and analyzed their essential attributes and operational characteristics. By carefully analyzing all the work above, it is not hard to find that resource and service sharing and enterprise collaboration taking the form of networks are the key for enterprises to stay competitive when confronting constantly changing market environment and customers' demands.

On the other hand, simulation has been widely adopted in manufacturing system design, operation, and simulation language/package development [13]. Cicirelli et al. [14] studied modeling and simulation of complex manufacturing 


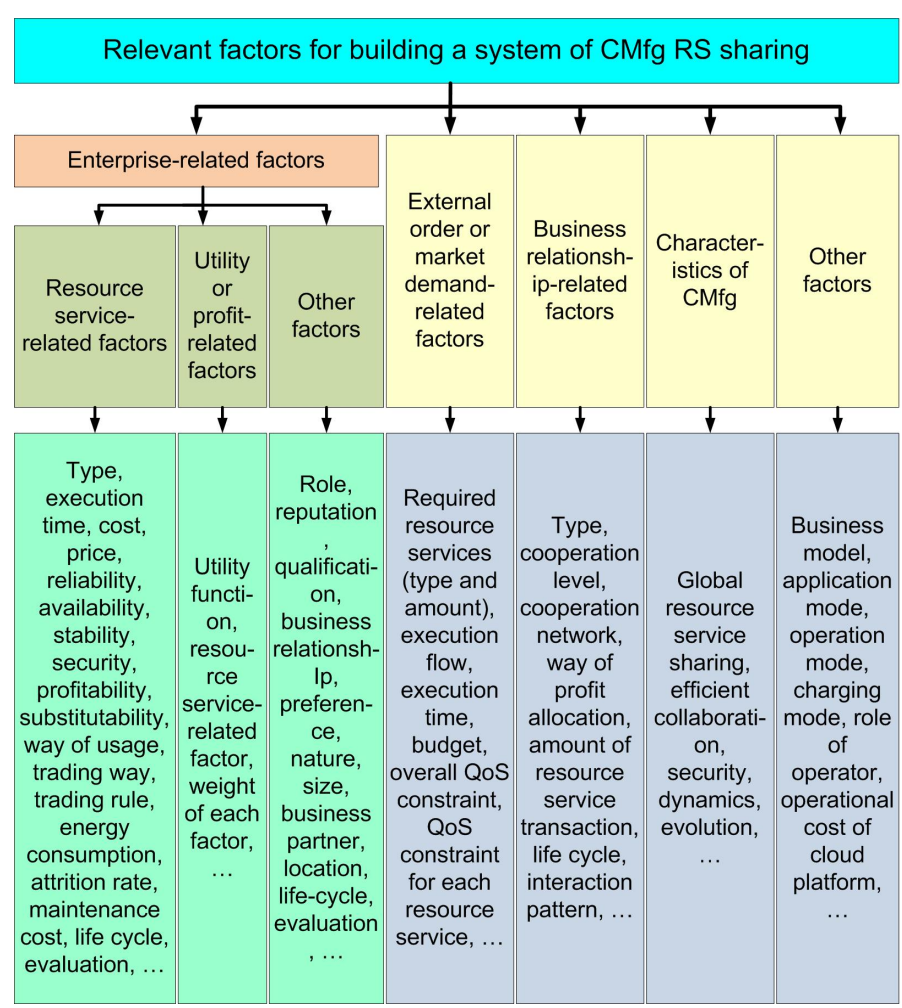

Fig. 1. Relevant factors for building a system of CMfg RS sharing

systems using statechart-based actors. Fowler and Rose [15] presented the challenges in modeling and simulation of complex manufacturing systems.

\section{Relevant factors for building a system of CMfg RS sharing}

In this section, we present the factors that are relevant for modeling a system of CMfg RS sharing, as shown in Fig. 1. According to the modeling requirements, the factors can be divided into five groups. 1) Enterprise-related factors. Because resource service sharing occurs among enterprises, it is natural and essential to consider the enterprise-related factors, which fall into three categories, resource service-related factors, utility or profit-related factors and other factors that do not belong to the previous two categories. 2) External order or market demandrelated factors. Resource service sharing among enterprises occurs in the course of fulfilling requirement tasks. 3) Business relationship-related factors. We consider them as a separate category because they do not belong to any enterprise. Different business relationships between enterprises can have different impacts 


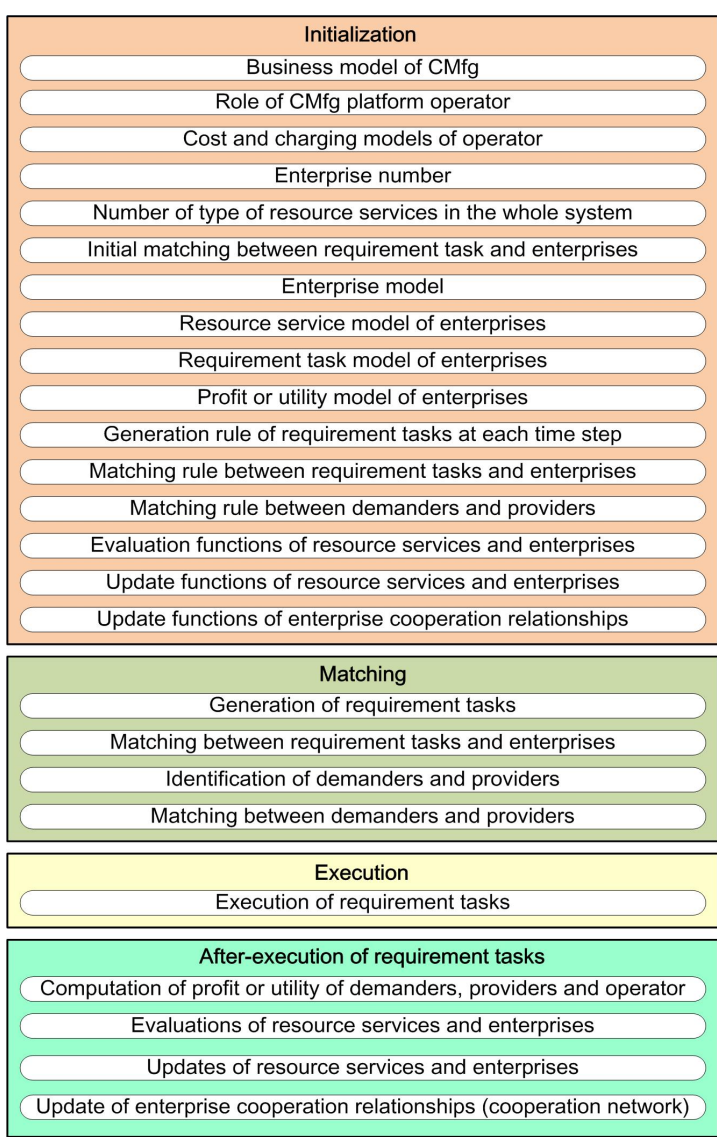

(a)

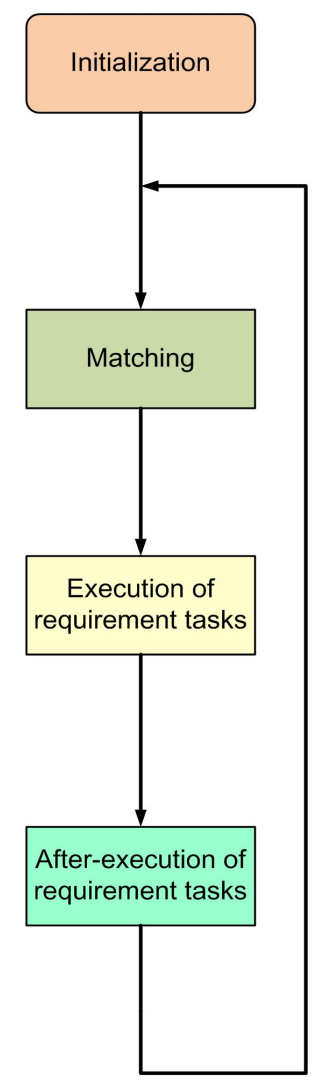

(b)

Fig. 2. (a) Activities in each procedure and (b) modeling procedure

on resource service sharing. 4) Characteristics of CMfg. The typical characteristics of CMfg that can influence resource service sharing should be considered. 5) Other factors. They are usually the macroscopic factors (such as business model and application mode) that need to be taken into account.

\section{Modeling procedure for a system of CMfg RS sharing}

This section describes the modeling procedure for a CMfg RS sharing system in detail (Fig.2).

\subsection{Initialization}

Much initialization work is needed, as shown in Fig.2. First, we need to deal with the business model. CMfg supports various business models such as general 
RS exchange, virtual enterprise, extended enterprise. Because in this paper, we focus on RS sharing among enterprises, and thus the business model is businessto-business (B2B). For the operator, apart from providing functional services to both demanders and providers (as an intermediary), it can even provide manufacturing services (as a provider). CMfg has some operational cost, and thus needs to charge enterprises to maintain the operation of the CMfg platform. The point here is to determine the billing functions according to the overall cost.

In order to acquire reliable research results, there should be a huge number of enterprises in a system of CMfg RS sharing. Building a model with a very small number of enterprises may result in erroneous results. For example, if the number is small, the essence of enterprise cooperation network (such as structure and dynamics) cannot be well captured. It is also an important problem as to whether consider the type of RSs. It is a common practice to assume several abstract types of RSs or just ignore it when RS type is not critical for research results.

Building a proper enterprise model is crucial for the success of building a system of CMfg RS sharing. We can assume that each enterprise has several types of RSs. For each RS, attributes such as cost, price, execution period, reliability and availability should be considered. In addition, an enterprise can also have one or several RTs. Each RT requires one or several types of RSs. For RTs, we can either explicitly consider the execution flow or ignor it when the execution flow does not essentially influence research results. Each RT also has an execution period, which can be set randomly or computed according to the required RSs and the execution flow. The budget of a RT as a constraint for selecting suitable RSs should also be considered.

Utility or profit as an important driving factor for RS sharing is also very critical. In CMfg, an enterprise can obtain some utility or profit by fulfilling a RT, alone or cooperatively. Profit is usually related to price and production cost, while utility can comprehensively describe the extent of satisfaction of an enterprise. For profit, we need to specify production cost and RS price, while utility, we need to determine the factors concerned (Fig.1) and then design a utility function.

In this paper, we assume the periodic business interactions among enterprises, which are driven by periodic RTs. Hence, how to generate RTs at each time step is an important issue. The number of RTs and the total amount of required RSs need to be considered. The following approach provides a feasible solution. At each time step, $N p_{t}$ RTs are generated, where $0<p_{t} \leq 1$ is a probability used for governing RT density, and $N$ is the number of enterprises in a CMfg system.

After the generation of RTs, the next step is to properly allocate them to the enterprises involved. In the real world, an enterprise which brings a higher utility to customers can win orders. Hence, the RT allocation is actually a process of preferential selection of the enterprises with high-quality RSs. The key is how to determine the related factors and the selection function.

RS supply-demand matching is a core issue in this modeling framework. Because in CMfg, there are a very large number of demanders and providers sub- 
mitting their requests and idle RSs simultaneously, it is a typical many-to-many matching problem. A successful matching depends on: (1) characteristics of RSs. RS type, cost, price, QoS (including reliability, availability, etc.) can affect the mutual selection between demanders and providers. (2) decision-making on RS sharing. In CMfg, an enterprise can choose to host a RT or participate in the RTs hosted by other enterprises. For a provider, hosting a RT is always preferable. However, when there is no RT, sharing the idle RS with the demanders can be profitable, which, however, decreases the likelihood for hosting a RT in the future (which usually is more profitable). In this case, the careful decision-making as to whether or how many RSs are shared is needed. (3) matching algorithm. There are primarily two solutions: developing a parallel algorithm to deal with all requirements simultaneously or adopting a serial algorithm that deal with the requirement successively. The former endows each enterprise with equal chance to access the RSs while in the latter case, the chance is different. Whatever the algorithm, the matching between demanders and providers is a mutual selection process. (4) RS transaction rule. It mainly refers to the self-defined rules by enterprises for the owned RSs. For example, a demander may have a specific requirement as to the required RSs in terms of QoS, price and location. (5) RS transaction manner. The real RS transaction process may take many forms, including negotiation, auction, etc. Game theory can be a powerful theoretical tool for the matching between demanders and providers $[8,10]$.

In order to foster an open and fair transaction environment, evaluation (including RS evaluation and enterprise evaluation) is essential. The key is to design proper evaluation functions. In CMfg, RSs and enterprises dynamical change over time [16]. The former includes improvement of RS QoS, elimination of RSs, generation of new RSs, etc., while the latter includes enterprise expansion, downsizing, elimination and generation, etc. For the process of RS or enterprise elimination, the key is to build a mathematical function, especially the function relation between maintenance (operation) cost and profit of RSs (enterprises). For the process of improving RS quality or purchasing new RSs, the key is to determine the function relation between RS improvement or purchasing cost and the investment. With the development of a CMfg system, based on the transaction of RSs, an enterprise cooperation network can be formed which evolves over time. Hence, the update includes the addition of new cooperation relationship, increase of cooperation weight, update of the amount of traded RSs, update of cooperation level and so forth. Complex network provides an effective approach for modeling the enterprise cooperation network [17].

\subsection{Matching}

There are two types of matching: matching between RTs and enterprises, and matching between providers and demanders. First, we need to generate RTs according to the generation rule, and then allocate them to enterprises. At this moment, some enterprises may have insufficient RS to fulfill their RT while others with few or no RTs have excess RSs. The former are referred to as demanders while the latter providers. Though sharing RTs and RSs, both demanders and 
providers can have a higher profit. The next step is to match demanders with providers according to the pre-defined matching algorithm. After matching, enterprises fulfill their RTs collaboratively.

\subsection{After-execution of RTs}

At the end of each time step, a certain RTs will be completed. At this moment, each enterprise computes its profit or utility based on the profit or utility function. The operator also computes its profit or utility. At the same time, demanders evaluate the shared RSs and demanders and providers can also mutually evaluate. A CMfg system may also have an evaluation system. Finally, an enterprise may also improve their RSs or eliminate the inferior RSs, whose maintenance cost is higher than the profit they bring. The enterprises that cannot make profits may also be eliminated from the system. According to the RS transaction, enterprises update their cooperation relationship, which can have important influence on future business interactions (e.g., partner selection).

\section{Conclusions}

In this paper, we propose a modeling framework for RS sharing in CMfg. The relevant factors are systematically summarized, and the procedure for modeling a system of CMfg RS sharing is presented.

RS sharing is one of the most important purposes of CMfg, which determines the performance and efficiency of a CMfg system. Hence, the research on methods and mechanisms of CMfg resource service sharing is significant, which, however, is challenged by the complexity of a CMfg system. In this regard, complex systems modeling and simulation provide a feasible and effective means. In order to apply this approach, we need to first find out the influencing factors for modeling a CMfg RS sharing system, as well as the basic modeling procedure. However, so far, there is no work directly dealing with such an important research issue. The current work, to some extent, bridges this gap and thus can provide some guidance for the future research on CMfg RS sharing.

Acknowledgments. This work is supported by the National Natural Science Foundation of China (NSFC) under grant Nos. 61203374, 61374199, 51475032, Natural Science Foundation of Beijing, China under grant No. 4142031, China Postdoctoral Science Foundation under grant Nos. 2012M520139 and 2013T60052, the Fundamental Research Funds for the Central Universities under grant No. JB140410.

\section{References}

1. Li, B.H., Zhang, L., Wang, S.L., Tao, F., Cao, J.W., Jiang, X.D., Song, X., Chai, X.D.: Cloud manufacturing: a new service-oriented networked manufacturing model. Computer Integrated Manufacturing Systems. 16, 1-7 (2010) 
2. Zhang, L., Luo, Y., Tao, F., Li, B.H., Ren, L., Zhang, X., Guo, H., Cheng, Y., Hu, A., Liu, Y.: Cloud manufacturing: a new manufacturing paradigm. Enterp. Inf. Syst. 8, 167-187 (2014)

3. He, W., Xu, L.: A state-of-the-art survey of cloud manufacturing. Int. J. Comput. Integ. M. 28, 239-259 (2015)

4. Xu, X.: From cloud computing to cloud manufacturing. Robot. Cim-Int. Manuf. 28, 75-86 (2012)

5. Tao, F., Cheng, Y., Zhang, L., Zhao, D.: Utility modelling, equilibrium, and coordination of resource service transaction in service-oriented manufacturing system. P. I. Mech. Eng. B-J. Eng. 226, 1099-1117 (2012)

6. Tai, D.Y., Xu, F.Y., Hu, W.: Cooperation concept and implementation of cloud manufacturing. Computer Integrated Manufacturing Systems. 18, 1575-1583 (2012)

7. Cheng, Y., Zhang, Y., Lv, L., Liu, J., Tao, F., Zhang, L.: Analysis of cloud service transaction in cloud manufacturing. In: 10th IEEE International Conference on Industrial Informatics (INDIN), pp. 320-325. IEEE Press, New York (2012)

8. Renna, P., Argoneto, P.: Capacity sharing in a network of independent factories: A cooperative game theory approach. Robot. Cim-Int. Manuf. 27, 405-417 (2011)

9. Yoon, S. W., Nof, S. Y.: Demand and capacity sharing decisions and protocols in a collaborative network of enterprises. Decis. Support Syst. 49, 442-450 (2010)

10. Renna, P., Argoneto, P.: Supporting capacity sharing in the cloud manufacturing environment based on game theory and fuzzy logic. Enterp. Inf. Syst. (ahead-ofprint), 1-18 (2014)

11. Moghaddam, M., Nof, S.Y.: Combined demand and capacity sharing with best matching decisions in enterprise collaboration. Int. J. Prod. Econ. 148, 93-109 (2014)

12. Jagdev, H. S., Thoben, K.D.: Anatomy of enterprise collaborations. Prod. Plan Control. 12, 437-451 (2001)

13. Negahban, A., Smith, J.S.: Simulation for manufacturing system design and operation: Literature review and analysis. J. Manuf. Syst. 33, 241-261 (2014)

14. Cicirelli, F., Furfaro, A., Nigro, L.: Modelling and simulation of complex manufacturing systems using statechart-based actors. Simul. Model Pract. Th. 19, 685-703 (2011)

15. Fowler, J.W., Rose, O.: Grand challenges in modeling and simulation of complex manufacturing systems. Simul.-T. Soc. Mod. Sim. 80, 469-476 (2004)

16. Liu, Y., Zhang, L., Tao, F., Wang, L.: Development and Implementation of Cloud Manufacturing: An Evolutionary Perspective. In: ASME 2013 International Manufacturing Science and Engineering Conference collocated with the 41st North American Manufacturing Research Conference, pp. V002T02A007. ASME Press, Madison, Wisconsin, USA (2013)

17. Albert, R., Barabási, A.L.: Statistical mechanics of complex networks. Rev. Mod. Phys. 74, 47 (2002) 